OPEN ACCESS

Edited by:

Paul Smith,

Connect Biopharma, United States

Reviewed by:

Qiang Xu,

Guangzhou University of Chinese

Medicine, China

Wenfeng Tan,

Nanjing Medical University, China

*Correspondence:

Huichao Wu

wuhuichao1011@126.com

Shouying Du

dushouying@outlook.com

Specialty section:

This article was submitted to

Pharmacogenetics and

Pharmacogenomics,

a section of the journal

Frontiers in Genetics

Received: 31 July 2021 Accepted: 15 October 2021

Published: 17 November 2021

Citation:

Zhu Y, Zhong W, Peng J, Wu H and Du S (2021) Study on the Mechanism of Baimai Ointment in the Treatment of

Osteoarthritis Based on Network Pharmacology and Molecular Docking

with Experimental Verification.

Front. Genet. 12:750681.

doi: $10.3389 /$ fgene.2021.750681

\section{Study on the Mechanism of Baimai Ointment in the Treatment of Osteoarthritis Based on Network Pharmacology and Molecular Docking with Experimental Verification}

\author{
Yingyin Zhu ${ }^{1}$, Wanling Zhong ${ }^{1}$, Jing Peng ${ }^{1}$, Huichao $W^{2 *}$ and Shouying $D u^{1 *}$ \\ ${ }^{1}$ School of Chinese Materia Medica, Beijing University of Chinese Medicine, Beijing, China, ${ }^{2}$ School of Traditional Chinese \\ Medicine, Beijing University of Chinese Medicine, Beijing, China
}

Purpose: The external preparation of the Tibetan medicine formula, Baimai ointment (BMO), has great therapeutic effects on osteoarthritis (OA). However, its molecular mechanism remains almost elusive. Here, a comprehensive strategy combining network pharmacology and molecular docking with pharmacological experiments was adopted to reveal the molecular mechanism of $\mathrm{BMO}$ against $\mathrm{OA}$.

Methods: The traditional Chinese medicine for systems pharmacology (TCMSP) database and analysis platform, traditional Chinese medicine integrated database (TCMID), GeneCards database, and DisGeNET database were used to screen the active components and targets of BMO in treating $\mathrm{OA}$. A component-target $(\mathrm{C}-\mathrm{T})$ network was built with the help of Cytoscape, and the Gene Ontology and Kyoto Encyclopedia of Genes and Genomes (KEGG) pathway enrichment through STRING. Autodock Tools which was used to dock the key components and key target proteins was analyzed. Animal experiments were performed to verify the key targets of BMO. Hematoxylin-eosin and toluidine blue staining were used to observe the pathology of joints. Protein expression was determined using enzyme-linked immunosorbent assay.

Results: Bioactive compounds and targets of $\mathrm{BMO}$ and $\mathrm{OA}$ were screened. The network analysis revealed that 17- $\beta$-estradiol, curcumin, licochalone A, quercetin, and glycyrrhizic acid were the candidate key components, and IL6, tumor necrosis factor (TNF), MAPK1, VEGFA, CXCL8, and IL1B were the candidate key targets in treating OA. The KEGG indicated that the TNF signaling pathway, NF-kB signaling pathway, and HIF-1 signaling pathway were the potential pathways. Molecular docking implied a strong combination

\footnotetext{
Abbreviations: ADME, absorption, distribution, metabolism, and excretion; AUC, area under the curve; ANOVA, analysis of variance; $\mathrm{BP}$, biological process; $\mathrm{BMO}$, Baimai ointment; $\mathrm{CC}$, cell composition; $\mathrm{C}-\mathrm{T}$, component-target; DL, drug likeness; EDTA, ethylenediaminetetraacetic acid; ELISA, enzyme-linked immunosorbent assay; GO, Gene Ontology; HE, hematoxylin-eosin; HL, half-life; HDON, hydrogen bond donor number; HACC, hydrogen bond acceptor number; KEGG, Kyoto Encyclopedia of Genes and Genomes; MF, molecular function; OA, osteoarthritis; OB, oral bioavailability; PBS, phosphate-buffered saline; PDB, Protein Data Bank; PPI, protein-protein interaction; SD, Sprague Dawley; TCMID, traditional Chinese medicine integrated database; TCMSP, traditional Chinese medicines for systems pharmacology database and analysis platform.
} 
between key components and key targets. The pathology and animal experiments showed BMO had great effects on OA via regulating IL6, TNF, MAPK1, VEGFA, CXCL8, and IL1B targets. These findings were consistent with the results obtained from the network pharmacology approach.

Conclusion: This study preliminarily illustrated the candidate key components, key targets, and potential pathways of $\mathrm{BMO}$ against $\mathrm{OA}$. It also provided a promising method to study the Tibetan medicine formula or external preparations.

Keywords: Baimai ointment, osteoarthritis, network pharmacology, molecular docking, molecular mechanism, external preparation

\section{INTRODUCTION}

Osteoarthritis $(\mathrm{OA})$ is a common chronic disease with cartilage degradation in the clinic, characterized by arthritis, synovial inflammation, joint edge, and underlying subchondral bone lesions (Krustev et al., 2015). Clinical manifestations include joint pain, swelling, stiffness, and even deformity with limited movement (Nüesch et al., 2011; Centers for Disease Control and Prevention, 2020). At present, the treatment of OA mainly includes oral drug therapy, intra-articular injection, exercise, and surgery (Sellam et al., 2019; Tao et al., 2020), which was reported to have the limitations of drug tolerability and side effects, inadequate symptom control, and invasive intervention (Xu et al., 2017; Kolasinski et al., 2020). Therefore, a noninvasive and effective treatment with better bioavailability and less systemic toxicity needs to be developed.

Tibetan medicine, the second largest traditional Chinese medicine system in China, has rich clinical applications in treating OA with its characteristic ingredients (Ren et al., 2018; Zhang et al., 2018; Fu et al., 2020). External preparation is a vital part of Tibetan medicine, which can avoid first-pass metabolism, deliver less side effects, and be noninvasive compared to other therapies. Baimai ointment (BMO) is a representative formula of Tibetan medicine for external use, which was first recorded in Clinical Notes of Tibetan Medicine in the 16th century with a long history, and has its origins in Four Medical Classics and other traditional Tibetan medicine classics (Gong et al., 2012; Zhang et al., 2018). Besides, BMO was officially recorded in the Drug Standards of Tibetan Medicine by the Ministry of Health of the People's Republic of China (Xing-ping et al., 2014). According to the theory of Tibetan medicine, OA belongs to Baimai disease (Ren et al., 2018), and BMO can treat OA with the effect of dispersing cold together with removing dampness, removing blood stasis, and soothing tendons by improving blood circulation (Liu et al., 2007). The effect of BMO in treating $\mathrm{OA}$ has been tested in clinical practice for hundreds of years. A randomized clinical trial of $124 \mathrm{OA}$ patients showed that BMO had significant advantages in reducing pain and recovering joint function by employing BMO combined with acupuncture (Wang and Li, 2019). Besides, BMO can be used to alleviate pain after knee replacement surgery (Dingzeng et al., 2020). Studies have shown that the mechanism of BMO in treating $\mathrm{OA}$ is related to inflammation and regulation of chondrocyte proliferation and apoptosis (Zhang et al., 2012; Jiang et al., 2019). However, the specific molecular mechanism is still unclear, which limits its further application. Therefore, an in-depth study has to be carried out to further illuminate the mechanism and give theoretical support for the clinical application of BMO.

Network pharmacology is a burgeoning research method for new drug design and development based on the theoretical progress of multidirectional pharmacology, systems biology, and omics (Li et al., 2019). The chemical compositions and active targets of Tibetan medicine are complex, which is consistent with the integrity of network pharmacology (Pang et al., 2018). The molecular docking technology refers to the simulated binding of ligands and receptor proteins via a computer platform (Ferreira et al., 2015). In this study, molecular docking was applied to test the binding ability of the key components with key targets. Furthermore, experimental pharmacological studies were employed to reveal the pharmacodynamic effects and mechanisms of BMO against $\mathrm{OA}$.

The research thoroughly illustrated the molecular mechanism of BMO against OA, which can promote the application and development of BMO and may result in more effective therapeutics for OA. Also, this article provided a comprehensive strategy to study the molecular mechanisms of external preparation and gave a new idea for the study of Tibetan medicine.

\section{MATERIALS AND METHODS}

\section{Reagents and Materials}

Baimai Ointment ${ }^{\circledR}$ was purchased from Qizheng Tibetan Medicine Corporation (Tibet, China). Diclofenac diethylamine Emulgel (Votalin ${ }^{\circledR}$ ) was purchased from Novartis Pharma Corporation (Beijing, China). The hematoxylin-eosin (HE) staining kit (C0105) was purchased from Beyotime Biotechnology (Shanghai, China). A toluidine blue staining kit (DA0059) was purchased from Leigen Biotechnology (Beijing, China). The enzyme-linked immunosorbent assay (ELISA) kits including CXCL8, IL-1 $\beta$, IL-6, MAPK1, tumor necrosis factor (TNF), and VEGFA were obtained from Meimian Biotechnology (Jiangsu, China). All other chemicals used in this study were of analytical grade. 


\section{Collection of Bioactive Compounds and Related Targets}

Firstly, the components of Curcumae longae Rhizoma, Zanthoxyli Pericarpium, Moschus, and Glycyrrhizae Radix et Rhizoma, the main ingredients of $\mathrm{BMO}$, were collected from the traditional Chinese medicines for systems pharmacology database and analysis platform (TCMSP) (http://tcmspw.com/) and traditional Chinese medicine integrated database (TCMID) (http://www.megabionet.org/tcmid/) (Wang et al., 2019; Cui et al., 2020). Drug likeness (DL) $\geq 0.18$ was set as the threshold to screen the bioactive components (Wang et al., 2015; Zeng et al., 2021). Since there are too many components in Glycyrrhizae Radix et Rhizoma, to facilitate the study, the absorption, distribution, metabolism, and excretion (ADME) parameters were added as follows: half-life (HL) $\geq 4 \mathrm{~h}$ (Yang et al., 2014), hydrogen bond donor number $(\mathrm{HDON})<5$, and hydrogen bond acceptor number (HACC) $<5$ (Lipinski et al., 2001). Secondly, the targets related to the obtained components were screened through the TCMSP database and GeneCards database (https://www.genecards.org/) (Chen and Sun, 2021), and the targets of the components that could not be retrieved in these databases were predicted by the TargetNet database (http://targetnet.scbdd.com/) with an area under the curve $\geq 7$ and prediction probability $=1$. Finally, the UniProt database (https://www.uniprot.org/) was used to correct the targets obtained to official names with selected species "Homo sapiens."

\section{Collection of Osteoarthritis Disease Targets}

The targets associated with OA disease were collected through the TCMSP database, GeneCards database, and DisGeNET database (http://www.disgenet.org/web/DisGeNET/menu/home/). After removing the repeated targets, they were corrected to official names with selected species "Homo sapiens" using the UniProt database.

\section{Component-Target Visualization Network Construction}

The network of components and related targets of BMO was constructed for visual analysis through Cytoscape 3.6.1 (https:// cytoscape.org/). In the network, the nodes with diverse colors and shapes were symbolled for different components and targets. According to the degree of nodes, the key compounds were screened via the C-T network.

\section{Screening of Candidate Targets and Construction of the Protein-Protein Interaction Network}

The overlapped genes related to $\mathrm{BMO}$ and $\mathrm{OA}$ disease were screened as candidate targets using Venny (http://bioinfogp.cnb. csic.es/tools/venny/). The candidate targets were inputted to STRING 11.0 (https://string-db.org/) which includes a great number of protein-protein interaction (PPI) relationships (von Mering et al., 2005). With the confidence score $>0.7$ as the screening condition, a PPI network was analyzed after removing the scattered targets. Then, the network information was input into Cytoscape 3.6.1 to visualize the PPI network.

\section{Gene Ontology and Kyoto Encyclopedia of Genes and Genomes Pathway Enrichment}

Gene Ontology (GO) functional and Kyoto Encyclopedia of Genes and Genomes (KEGG) pathway enrichment analyses were conducted with the overlapped targets using the STRING database. According to the biological process (BP), cell composition (CC), molecular function (MF), and the actual roles of the pathways with $p$ value $<0.05$, we speculated and analyzed the main molecular biological processes or signal pathways of BMO in the treatment of OA. Then, a bubble chart was drawn online using imageGP (http://www.ehbio. com/ImageGP/).

\section{Molecular Docking}

The key components and key targets were obtained from the component-target (C-T) network and PPI network, respectively, for molecular docking. Firstly, the three-dimensional (3D) molecular structures of the core components and chemical drugs used for treating OA were downloaded from the PubChem database (https://pubchem.ncbi.nlm.nih.gov/) in the sdf format, then Chem3D software (https://www.chemdraw.com. $\mathrm{cn} /$ ) was used to convert them to pdb formats. The 3D structures of core target proteins were downloaded from the Protein Data Bank database (http://www.rcsb.org/) in pdb formats. Secondly, ligands and proteins were prepared through PyMOL (https:// pymol.org/) involving charge addition, atomic type assignment, water removing, and extraction of original ligands and ions. Then, the ligands and proteins were saved in the pdbqt format using AutoDock Tools 1.5.6 (http://autodock.scripps.edu/ ).Thirdly, AutoDock Tools was used for docking after setting grid box parameters. For each ligand, the lowest binding energy was selected as the result of molecular docking. Lastly, the docking conformation analysis and mapping were carried out by PyMOL software, and PLIP (https://projects.biotec.tudresden.de/plip-web/plip) was used to analyze the force between ligands and proteins (Yan and Zhou, 2021).

\section{Establishment of Rat Osteoarthritis Model and Drug Administration}

The Sprague Dawley (SD) rats used here were obtained from Vital River Laboratory Animal Technology Co., Ltd., (Beijing, China). All animal experimental procedures were approved by the Animal Ethics Committee of Beijing University of Chinese Medicine (BUCM-4-2019101701-4001). 24 SD rats were randomly divided into four groups, namely, sham-operated group, model group, positive control group, and BMO group. We used a modified Hulth method to establish the rat OA model (Zhou et al., 2018). Firstly, the animals were denied food and water for $8 \mathrm{~h}$ before the operation and were fixed in the supine position on the operating table after being anesthetized with ketamine $(100 \mathrm{mg} / \mathrm{kg})$ and xylazine $(10 \mathrm{mg} / \mathrm{kg})$ by intraperitoneal injection, and applied a tourniquet to the proximal right leg. 
TABLE 1 | Modified Mankin scoring criteria.

\begin{tabular}{|c|c|c|}
\hline Organization structure & Microscopic features & Scores \\
\hline \multirow[t]{4}{*}{ Cartilage structure } & Normal & 0 \\
\hline & The surface is slightly worn, regular arrangement & 1 \\
\hline & Hierarchy disorder, irregular arrangement & 2 \\
\hline & The surface is seriously worn, irregular arrangement & 3 \\
\hline \multirow[t]{4}{*}{ Toluidine blue staining } & Normal & 0 \\
\hline & Mild decrease & 1 \\
\hline & Moderate decrease & 2 \\
\hline & Severe decrease & 3 \\
\hline \multirow[t]{4}{*}{ Tide line } & Structure of tidal lines is intact & 0 \\
\hline & Multilevel structure & 1 \\
\hline & Tidal blur & 2 \\
\hline & There are blood vessels through the tidal line & 3 \\
\hline \multirow[t]{4}{*}{ Proportion of hyaline cartilage and calcified cartilage } & Normal & 0 \\
\hline & Mild decrease & 1 \\
\hline & Moderate decrease & 2 \\
\hline & Severe decrease & 3 \\
\hline
\end{tabular}

Secondly, the rats were shaved, and a $4-\mathrm{cm}$ incision was made in the median knee. Then, the medial collateral ligament and medial meniscus of the rats' right knee were cut in all groups except in the sham-operated group, and in sham-operated group, we only opened the joint cavity without destroying any joint structure. Next, the articular cavity and incision were flushed with physiological saline and sutured using a 3-0 absorbable suture. The contralateral knee was placed in the extension position with a plaster after the operation. Penicillin was intramuscularly injected with a dose of $20 \mathrm{U}$ per day to prevent infection within 3 days after the operation.

After operation, BMO and Votalin Emulgel were applied to the right knee joint of the rats in the BMO group and positive control group, respectively, followed by massage for $5 \mathrm{~min}$. The dosage of the ointment was $1 \mathrm{~g}$ per time, twice a day. The period of administration was 4 weeks.

\section{Hematoxylin-Eosin and Toluidine Blue Staining}

After 4-week administration, the right knees of all rats were cut and put into $4 \%$ paraformaldehyde for fixation, then routinely decalcified with ethylenediaminetetraacetic acid. After being washed, the knee joints were dehydrated by step gradient ethanol and hyalinized by dimethylbenzene. Next, the joints were paraffin-embedded and sliced into $5-\mu \mathrm{m}$ sections. Neutral gum sealing was performed after conventional $\mathrm{HE}$ and toluidine blue staining, and light microscopy used to observe the slices. The modified Mankin score (Table 1) was used to evaluate the cartilage destruction (Mankin et al., 1981).

\section{Enzyme-Linked Immunosorbent Assay}

After 4-week administration, the rats in all groups were anesthetized and fixed, and their skin and subcutaneous tissues cut to expose the joint capsule. Then, $400 \mu$ l sterile phosphate-buffered saline was injected into the joint cavity. After repeatedly moving the joint, $300 \mu \mathrm{l}$ joint fluid was carefully extracted for ELISA, which was diluted and added according to the ELISA kit operating instructions. Firstly, it was incubated at $37^{\circ} \mathrm{C}$ for $30 \mathrm{~min}$, and the concentrated solution was washed thoroughly after dilution. It was then treated to enzymes, warm bath, washing, and color rendering and the termination liquid added to terminate the reaction. CXCL8, IL-1 $\beta$, IL-6, MAPK1, TNF, and VEGFA were measured within $15 \mathrm{~min}$ after the addition of the stop solution. Absorbance was measured immediately at $450 \mathrm{~nm}$ using an enzyme marker (Biotech, America), and a standard curve was derived to read the contents of IL-1 $\beta$, TNF, MAPK1, IL-6, CXCL8, and VEGFA in the joint fluid in different groups.

\section{Statistical Analysis}

Data were presented as the mean \pm standard deviation with at least six independent experiments. SPSS 20.0 software was used to perform statistical analyses by one-way analysis of variance, and $p<0.05$ was considered statistically significant.

\section{RESULTS}

\section{Active Components of Baimai Ointment}

A collection of 44 potential compounds were collected from TCMSP and TCMID databases according to certain criteria, including 7 in Curcumae longae Rhizoma, 8 in Zanthoxyli Pericarpium, 7 in Moschus, and 22 in Glycyrrhizae Radix et Rhizoma (Table 2).

\section{Component-Target Network Analysis}

A total of 874 targets corresponding to compounds in BMO were collected from TCMSP, GeneCards, and TargetNet databases, and the remaining 333 targets collected after duplicate removal. The C-T network constructed with Cytoscape 3.6.1 included 381 nodes and 917 edges (Figure 1). The purple rectangles represent the 333 corresponding targets of compounds. The hexagons in yellow, pink, blue, and red stand for compounds in Curcumae longae Rhizoma, Zanthoxyli Pericarpium, Moschus, and Glycyrrhizae Radix et Rhizoma, respectively. The green ellipse stands for Curcumae longae Rhizoma, Zanthoxyli Pericarpium, Moschus, and Glycyrrhizae Radix et Rhizoma. The size and 
TABLE 2 | Active compounds of BMO. A. Active compounds of Curcumae longae Rhizoma, Zanthoxyli Pericarpium, and Moschus.

\begin{tabular}{|c|c|c|c|c|}
\hline Herb name & Mol ID & Molecule name & MW & DL \\
\hline \multirow[t]{7}{*}{ Curcumae longae Rhizoma } & MOL000449 & Stigmasterol & 412.77 & 0.76 \\
\hline & MOL000493 & Campesterol & 400.76 & 0.71 \\
\hline & MOL000953 & CLR & 386.73 & 0.68 \\
\hline & MOL000892 & TNP00001 & 368.41 & 0.41 \\
\hline & MOL000951 & Dihydrocurcumin & 370.43 & 0.41 \\
\hline & MOL000946 & Demethoxycurcumin & 338.38 & 0.33 \\
\hline & MOL000945 & Bisdemethoxycurcumin & 308.35 & 0.26 \\
\hline \multirow[t]{8}{*}{ Zanthoxyli Pericarpium } & MOL013271 & Kokusaginin & 259.28 & 0.20 \\
\hline & MOL002663 & Skimmianin & 259.28 & 0.20 \\
\hline & MOL002881 & Diosmetin & 300.28 & 0.27 \\
\hline & MOL000358 & beta-Sitosterol & 414.79 & 0.75 \\
\hline & MOL004368 & Hyperin & 464.41 & 0.77 \\
\hline & MOL005093 & Diosmin & 608.60 & 0.66 \\
\hline & MOL000514 & Nonacosane & 408.89 & 0.39 \\
\hline & MOL000098 & Quercetin & 302.25 & 0.28 \\
\hline \multirow[t]{7}{*}{ Moschus } & MOL010919 & 17-beta-Estradiol & 272.42 & 0.32 \\
\hline & MOL002359 & 6-Hydroxy-musizin-8-O-beta-D-glucoside & 394.41 & 0.50 \\
\hline & MOL000987 & Cholesterol & 384.76 & 0.67 \\
\hline & MOL002442 & Cholesteryl ferulate & 562.91 & 0.63 \\
\hline & MOL000737 & Morin & 302.25 & 0.27 \\
\hline & MOL007216 & n-Nornuciferine & 281.38 & 0.36 \\
\hline & MOL001232 & TES & 288.47 & 0.35 \\
\hline
\end{tabular}

B. Active compounds of Glycyrrhizae Radix et Rhizoma.

\begin{tabular}{|c|c|c|c|c|}
\hline Herb name & Mol ID & Molecule name & MW & DL \\
\hline & MOL000211 & Mairin & 456.78 & 0.78 \\
\hline & MOL000359 & Sitosterol & 414.79 & 0.75 \\
\hline & MOL003896 & 7-Methoxy-2-methyl isoflavone & 266.31 & 0.20 \\
\hline & MOL000392 & Formononetin & 268.28 & 0.21 \\
\hline & MOL004805 & Shinflavanone & 390.51 & 0.72 \\
\hline & MOL004835 & Glypallichalcone & 284.33 & 0.19 \\
\hline & MOL004838 & Kanzonol U & 308.35 & 0.38 \\
\hline & MOL004891 & Shinpterocarpin & 322.38 & 0.73 \\
\hline & MOL004910 & Glabranin & 324.40 & 0.31 \\
\hline & MOL004941 & N1793 & 256.27 & 0.18 \\
\hline & MOL004945 & Isobavachin & 324.40 & 0.32 \\
\hline & MOL004996 & Gadelaidic acid & 310.58 & 0.20 \\
\hline & MOL005003 & Licoagrocarpin & 338.43 & 0.58 \\
\hline & MOL005018 & Xambioona & 388.49 & 0.87 \\
\hline
\end{tabular}

transparency of the nodes were proportional to the degree. In the network, each compound interacted with 19.86 targets on average, and each target interacted with 2.62 compounds on average. Therefore, the phenomenon that one compound corresponds to multiple targets and one target corresponds to multiple compounds simultaneously exist, which reflects that the traditional Tibetan medicine treats diseases through the mechanism of multicomponent and multi-target. Furthermore, higher degrees indicate more essential nodes in the network. Quercetin, 17-beta-estradiol, and licochalcone A indicated high degrees, and thus might play chief roles in the effect of BMO treating OA. In our previous study, we found that curcumin and glycyrrhizic acid were also involved in the treatment of OA in BMO (Liang et al., 2019).

\section{Overlapped Targets and Protein-Protein Interaction Network Analysis}

After deleting repeated values and transferring to standard gene symbols, 2056 targets associated with OA were obtained from TCMSP, GeneCards, and DisGeNET databases. Among the 333 compounds and 2054 OA-related targets, there were 169 overlapped targets which were considered potential targets for subsequent studies (Figure 2A). The 169 related targets were 


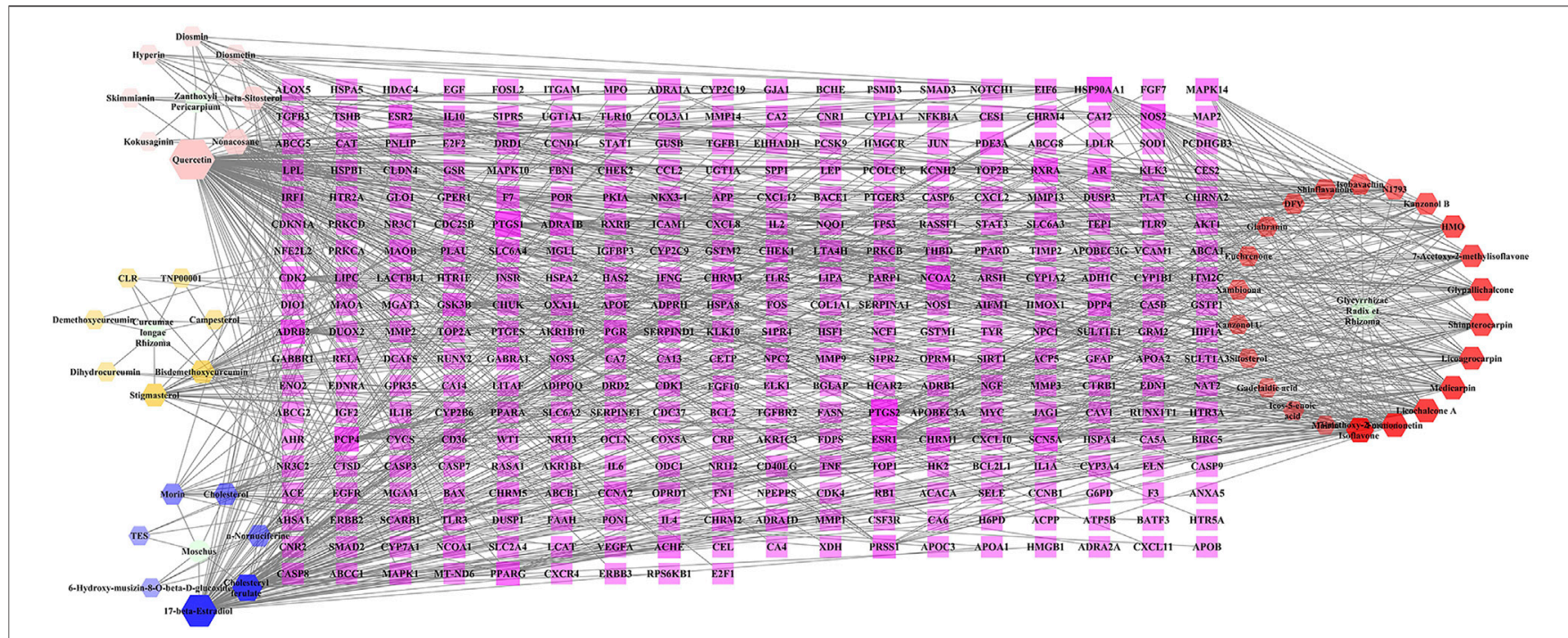

FIGURE 1 |C-T network of BMO. The purple rectangles represented 333 potential target genes of compounds. The hexagons in yellow, pink, blue, and red stand for compounds in Curcumae longae Rhizoma, Zanthoxyli Pericarpium, Moschus, and Glycyrrhizae Radix et Rhizoma, respectively. The green ellipse stands for Curcumae longae Rhizoma, Zanthoxyli Pericarpium, Moschus, and Glycyrrhizae Radix et Rhizoma. The size and transparency of the nodes were proportional to the degree.

input to STRING 11.0 and Cytoscape to analyze and construct a PPI network after removing scattered targets. As shown in Figure 2B, the PPI network included 162 nodes. Larger and red nodes indicated higher degrees. The nodes with the top 20 degrees are shown in Figure 2C, among which the most related targets with high degrees are IL6, TNF, MAPK1, VEGFA, CXCL8, and IL1B. These targets might play a crucial role in the PPI network in treating OA.

\section{Gene Ontology Analysis}

STRING 11.0 was used to analyze the BP, CC, and MF annotations of the 169 potential targets. There were $2082 \mathrm{GO}$ terms $(p<0.05)$, which included 1804 of BP, 183 of MF, and 95 of CC. Most of the BP terms were related to response to inflammatory, regulation of cytokines, cell proliferation, apoptosis, etc., and the main terms of MF were associated with protein binding, receptor binding, cytokine binding, etc. CC enrichment was mainly involved in the cytoplasm, endoplasmic reticulum, organelles, etc.

The GO terms related to OA could be classified into antiinflammatory, immunomodulatory, cellular regulation, and metabolism aspects, including 238 of BP and 11 of MF. There were inflammatory response, leukocyte migration, regulation of leukocyte, cell-cell adhesion, regulation of leukocyte activation, interleukin-1 receptor binding, TNF receptor binding, etc. The top 20 terms of BP and CC ranked according to the $p$-value, and 11 terms of MF are shown in Figure 3.

\section{Kyoto Encyclopedia of Genes and Genomes Analysis}

The KEGG pathway annotation indicated that 159 pathways $(p<$ $0.05)$ were enriched, mainly covering diseases, inflammation, immunity, metabolism, and cell regulation. Ranked according to the $p$-value, the top five pathways were pathways in cancer $(p=$ $\left.5.42 \mathrm{E}^{-32}\right)$, AGE-RAGE signaling pathway in diabetic complications $\left(p=2.65 \mathrm{E}^{-29}\right)$, TNF signaling pathway $(p=$ $\left.5.85 \mathrm{E}^{-18}\right)$, IL-17 signaling pathway $\left(p=1.04 \mathrm{E}^{-17}\right)$, and hepatitis $\mathrm{B}\left(p=1.04 \mathrm{E}^{-17}\right)$.

On further analysis, there were 50 pathways related to OA, among which 16 pathways were associated with antiinflammatory, 14 with immunomodulatory, and 20 with cellular metabolism. The KEGG pathways in the top 20 were related to OA with low $p$-values, as shown in Figure 4. The results suggested that the TNF signaling pathway, NF- $\kappa \mathrm{B}$ signaling pathway, and HIF-1 signaling pathway played a significant role in treating $\mathrm{OA}$ with $\mathrm{BMO}$.

\section{Molecular Docking}

Molecular docking was conducted to verify the binding ability of IL6, TNF, MAPK1, VEGFA, CXCL8, and IL1B with quercetin, 17-beta-estradiol, licochalcone A, curcumin, and glycyrrhizic acid according to network analysis and preliminary research. Meanwhile, chemical drugs used clinically to treat OA such as meloxicam, celecoxib, and nabumetone were also docked with selected target proteins as a reference. The results of the binding energy are shown in Table 3.

The results suggested that the binding energy ranged from $-0.71 \mathrm{kcal} \cdot \mathrm{mol}^{-1}$ to $-5.42 \mathrm{kcal} \cdot \mathrm{mol}^{-1}$. Besides, the binding energy was all less than 0 and less than $-3.0 \mathrm{kcal} \cdot \mathrm{mol}^{-1}$ accounted for $46.67 \%$. When the binding energy was less than 0 , it indicated that the ligands could bind to the target proteins freely. The binding energy of key components with key target proteins was equivalent or low compared to that of the chemical drugs, which indicated that the key components of BMO had better binding activity with key targets, thus verifying the reliability of the results of network pharmacology research to a certain extent. The binding energy of 17 - $\beta$-estradiol with the aforementioned target proteins was even 

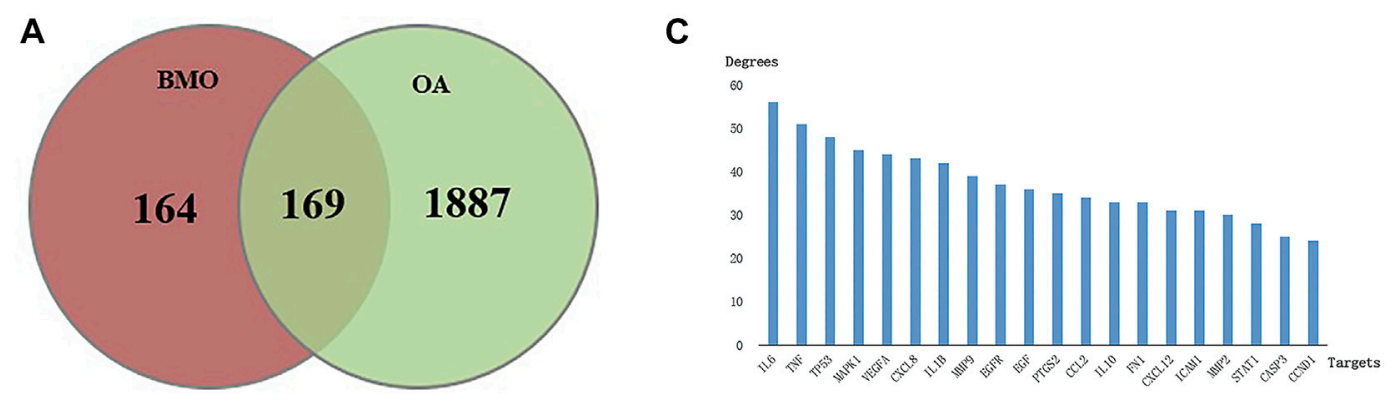

B

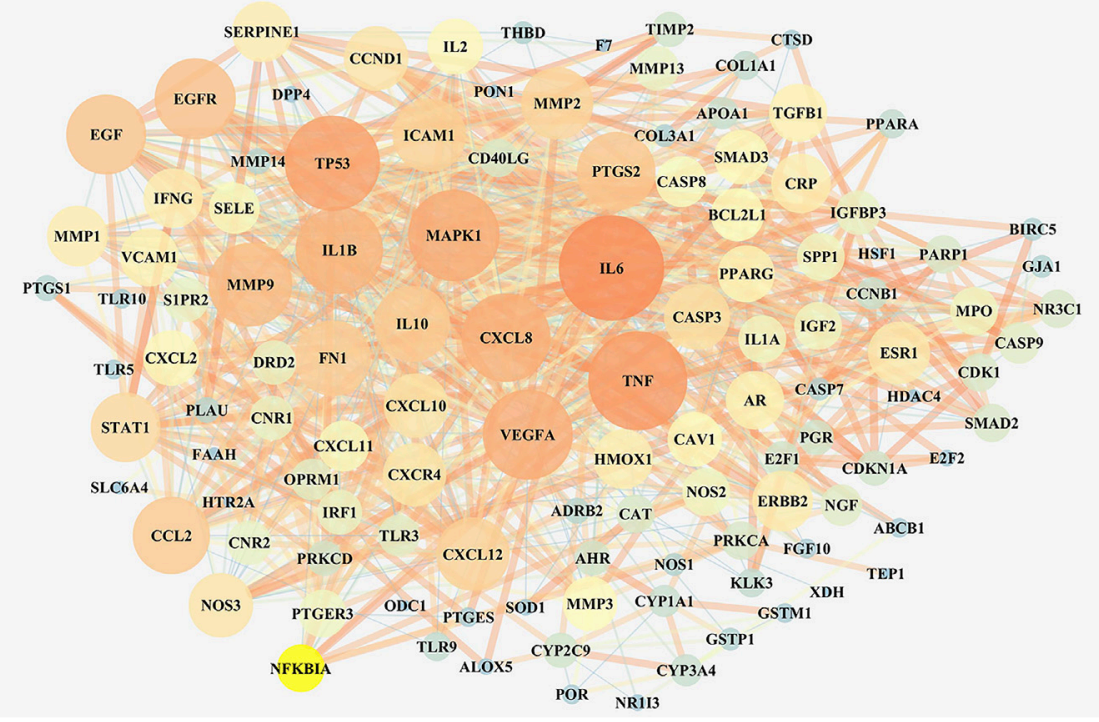

FIGURE 2 | Analysis of key targets of BMO in treating OA. (A) After obtaining 333 targets of BMO and 2056 targets of OA, we overlapped the two parts of targets and got 169 mutual targets. (B) PPI network of the 169 overlapped targets was analyzed and constructed by STRING and Cytoscape. Larger and red nodes indicate higher degrees. (C) Top 20 targets with highest degrees of BMO against OA.

lower than that of the chemical drugs. These components could interact with the key target proteins such as IL6, TNF, MAPK1, VEGFA, CXCL8, and IL1B to have an effect on OA.

The docking conformation of the five key components and their best combined target proteins are shown in Figures 5B-F, respectively. As shown in Figure 5A, 17-beta-estradiol could form a structure of hydrogen bond with Gly230 in MAPK1 whose length was 2.27, and the benzene ring in the compound could be $\mathrm{p}-\pi$ conjugated with the pentane ring in His 239 . As shown in Figure 5B, curcumin could form a structure of three hydrogen bonds with Arg6, Lys11, and Glu48 in CXCL8, and their bond lengths were $2.20,3.04$, and 1.98, respectively. As shown in Figure 5C, licochalcone A could form a structure of two hydrogen bonds with Met20 and Lys63 in IL1B, and their bond lengths were 1.94 and 2.50, respectively. As shown in Figure 5D, quercetin could form a structure of one hydrogen bond with Met20, two hydrogen bonds with Val41, and two hydrogen bonds with Lys63 in IL1B and their bond lengths were $2.62,2.19,2.06,3.00$, and 2.50 , respectively. As shown in Figure 5E, glycyrrhizic acid could form a structure of three hydrogen bonds with Leu25, Ser44, and Arg68 in CXCL8 and their bond lengths were $1.92,2.94$, and 2.86 , respectively. Besides, it could form a salt bridge with Arg68 in CXCL8, which enhanced the stability of binding.

In conclusion, there was a stable interaction between key compounds and key target proteins with different bonds. The results illustrated that the key compounds could bind to the active site of key target proteins to have an effect on treating OA.

\section{Pathology}

The knee joints of rats in each group were observed by HE and toluidine blue staining (Figure 6A). In the sham-operated group, the meniscus could be seen on the knee joint of rats in the medial side, and the cartilage surface was neat without cartilage wear and rupture. The cartilage was thick, and the chondrocytes were arranged in order. The cartilage of the tibial plateau could be divided into hyaline cartilage above and calcified cartilage below. Toluidine blue staining was clear. In the model group, the meniscus of the knee joint was lost, and the cartilage surface was seriously worn with its layer thinner. The arrangement of chondrocytes was disordered, and the tide line was not clear. The proportion of the hyaline cartilage and calcified cartilage 


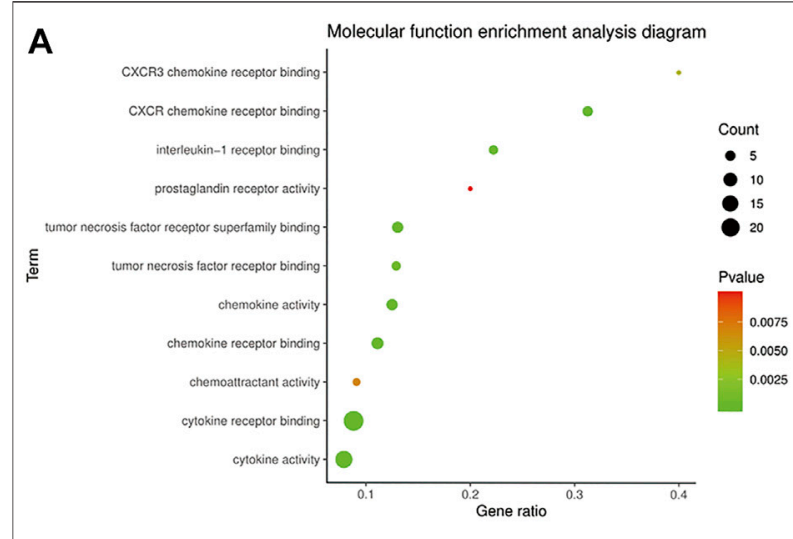

B
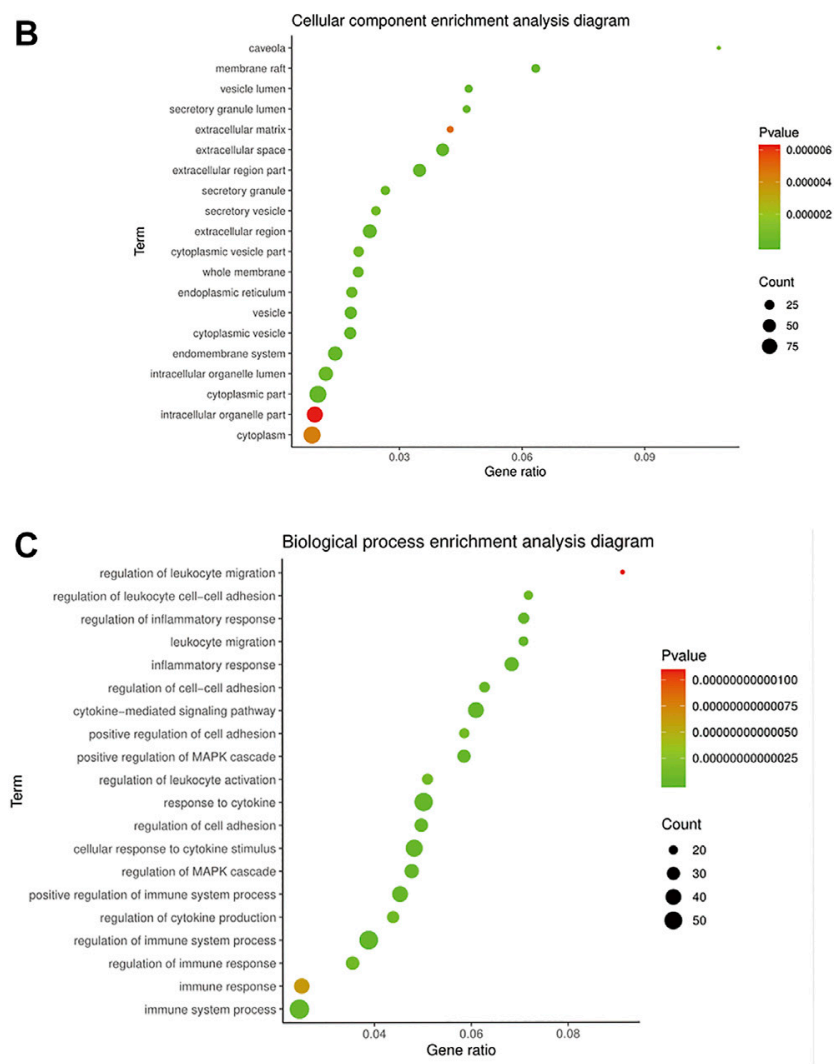

FIGURE 3 | GO enrichment of BMO in the treatment of OA. (A) 11 of $183 \mathrm{MF}$ terms related to the mechanism of OA. (B,C) Top 20 of CC and BP terms related to the mechanism of OA ranked according to the $p$-value.

decreased, and toluidine blue staining of the cartilage tissue was seriously depigmented, which indicated that the OA model was successfully established. Compared with the model group, the positive control group and BMO group were all in better conditions. The cartilage surface was slightly worn, and its layer was thicker than the model group. After treating with drugs, the chondrocytes arranged well, and the tidal line was clearly visible. The ratio of the hyaline cartilage to calcified cartilage increased, and toluidine blue staining was clear on the surface of the cartilage tissue. By comparing the modified
Mankin score of each group (Figure 6B), we found that there were significant differences $(p<0.001)$ between the shamoperated group and model group. After administration, the scores decreased significantly $(p<0.001)$. There was no significant difference between the positive control group and BMO group $(p>0.05)$.

\section{Expression of CXCL8, IL-1 $\beta$, IL-6, MAPK1, TNF, and VEGFA in Joint Fluid}

The levels of CXCL8, IL-1 $\beta$, IL-6, MAPK1, TNF, and VEGFA in joint fluid were detected by ELISA. As shown in Figure 7, compared to the sham-operated group, the levels of those cytokines both increased significantly $(p<0.001)$ in the model group, indicating the successful establishment of the OA model. After administration, the levels of those cytokines decreased significantly $(p<0.001)$ in both the BMO group and positive control group, which suggested BMO had effects on treating OA to a certain extent by regulating CXCL8, IL-1 $\beta$, IL-6, MAPK1, TNF, and VEGFA in the joint fluid.

\section{DISCUSSION}

Among all kinds of arthritis, $\mathrm{OA}$ is the most common one, which is called degenerative joint disease or wear and tear arthritis as well. The lesions of the disease that occur most frequently are in the knees, hips, and hands. OA is usually treated with combined therapies including medications, surgery, and exercise (Fortin et al., 2002; Totlis et al., 2021). Medication therapy is of most importance in treating $\mathrm{OA}$, which includes nonsteroidal antiinflammatory drugs, cartilage protectants, and intra-articular injection drugs in Western medicine (Lane and Thompson, 1997; Petrella and Wakeford, 2015). Compared to Western medicine that treats with a single target, traditional medicine such as traditional Tibetan medicine has its unique regulation effects with multi-target and multi-pathway, which overcome the shortages of drug resistance and unilateral effects (Liu et al., 2019; Yu et al., 2020).

$\mathrm{BMO}$ is a famous formula for external use in treating OA, which consists of 11 traditional Tibetan medicines such as Curcumae longae Rhizoma, Zanthoxyli Pericarpium, Moschus, Glycyrrhizae Radix et Rhizoma, Myristicae Semen, Kaempferia galanga Linn, and so on (Gong et al., 2012; Xing-ping et al., 2014). According to the traditional theory of Tibetan medicine and reported research, Curcumae longae Rhizoma, Zanthoxyli Pericarpium, Moschus, and Glycyrrhizae Radix et Rhizoma were considered the main ingredients of BMO against OA with their anti-inflammation and other pharmacological actions. Curcumae longae Rhizoma is the sovereign medicine of BMO, which has the effect of blood-breaking and promoting Qi, dredging channels, and relieving pain. Curcumin is the major and effective component of Curcumae longae Rhizoma, which is applied as a nonsteroidal anti-inflammatory drug in the clinic and has a significant effect on OA (Buhrmann et al., 2011). Zanthoxyli Pericarpium and Moschus belong to warm-hot natured drugs, which possess the functions of promoting blood circulation and 


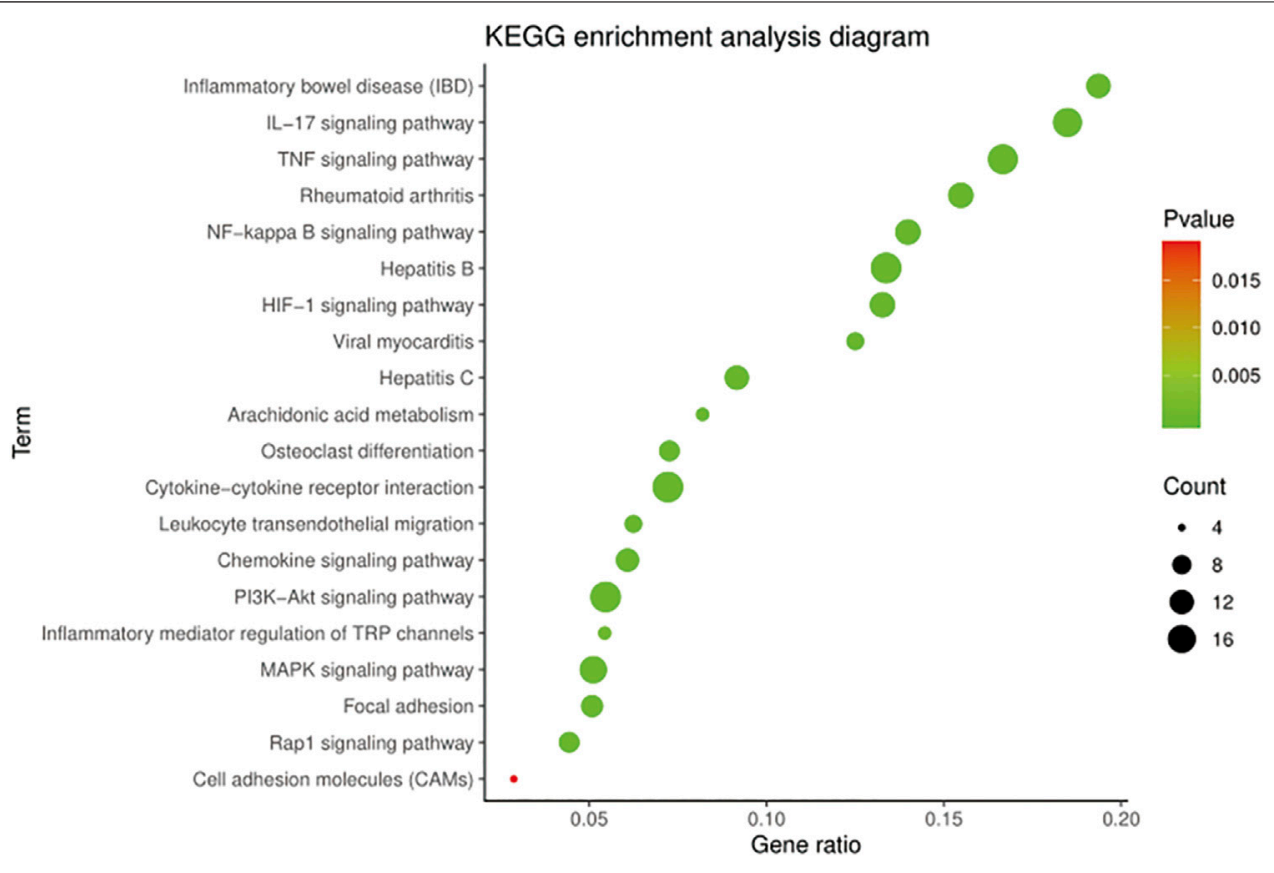

FIGURE 4 | KEGG enrichment of BMO in the treatment of OA. 159 KEGG pathways $(p<0.05)$ were enriched, among which 50 pathways were related to OA. Top 20 of the 50 pathways with the low $p$-value are shown.

TABLE 3 | Binding energy of key components of BMO with key targets.

Components and

chemicals

17-beta-estradiol

Curcumin

Licochalcone A

Quercetin

Glycyrrhizic acid

Meloxicam

Celecoxib

Nabumetone

\begin{tabular}{ccc}
\hline IL6 & TNF & MAPK1 \\
-3.75 & -4.76 & -5.42 \\
-1.83 & -3.57 & -3.52 \\
-2.28 & -3.14 & -2.37 \\
-1.90 & -2.63 & -2.27 \\
-2.56 & -2.87 & -2.92 \\
-3.26 & -3.91 & -3.44 \\
-2.97 & -3.57 & -3.24 \\
-3.06 & -3.86 & -2.81
\end{tabular}

Binding energy (kcal.mol-1)

relieving pain (Wang et al., 2014; Kim et al., 2019). Besides, Glycyrrhizae Radix et Rhizoma has been widely reported to have anti-inflammatory and antioxidant effects, etc. (Feng et al., 2013; Tavvafian et al., 2020).

However, the molecular mechanism of BMO has not been clarified. In this study, we adopted a network pharmacology approach together with molecular docking and experimental validation to investigate the bioactive compounds and molecular mechanisms of BMO in treating OA. From the network pharmacology studies, bioactive compounds and gene targets of BMO were obtained, and 169 intersection targets of BMO and OA were screened. Combined with C-T network analysis and previous studies (Liang et al., 2019), glycyrrhizic acid, 17- $\beta$-estradiol, curcumin, licochalone $A$, and quercetin were considered the candidate key components, and IL6, TNF, MAPK1, VEGFA, CXCL8, and IL1B were the candidate key targets in treating OA. The KEGG indicated that the TNF signaling pathway, NF- $\kappa \mathrm{B}$ signaling pathway, and HIF-1 signaling pathway were the potential pathways. Molecular docking implied a strong combination between key components and key targets. The pathology and animal experiments showed $\mathrm{BMO}$ had a great effect on OA via regulating IL6, TNF, MAPK1, VEGFA, CXCL8, and IL1B targets. These findings are consistent with the results obtained from the network pharmacology approach.

Through network pharmacology research, a total of 44 potential compounds were screened according to the ADME parameters such as DL, HL, HDON, and HACC. To further analyze, a C-T network was constructed to show the interaction between compounds and targets, among which 17- $\beta$-estradiol, licochalcone $\mathrm{A}$, and quercetin showed higher degrees. The higher the degree value, the more targets the compounds will interact with. Our previous study has shown that glycyrrhizic acid and curcumin were the major effective compounds of BMO which 


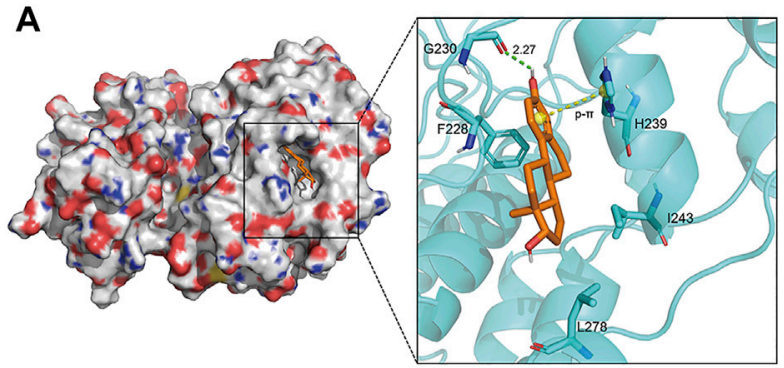

C

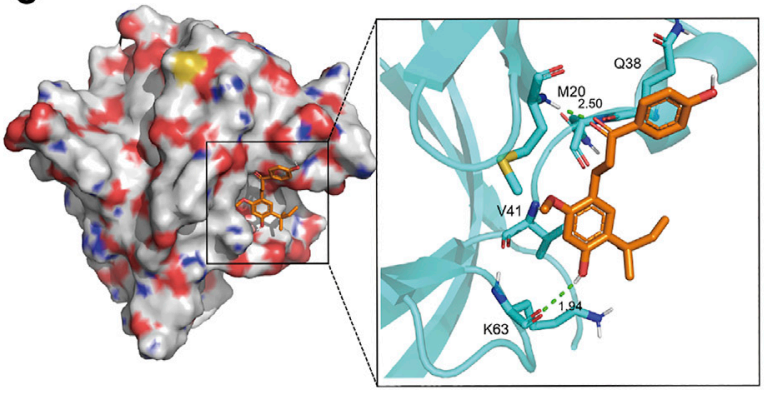

B
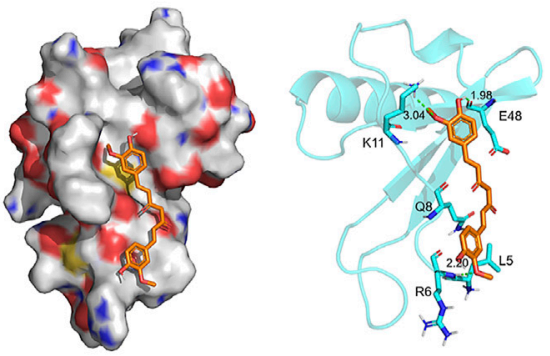

D

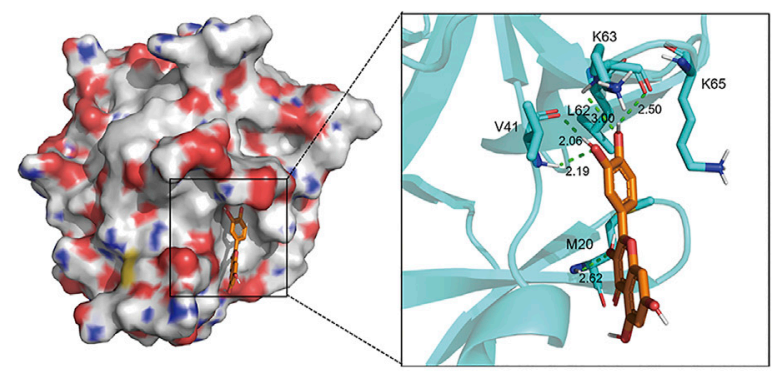

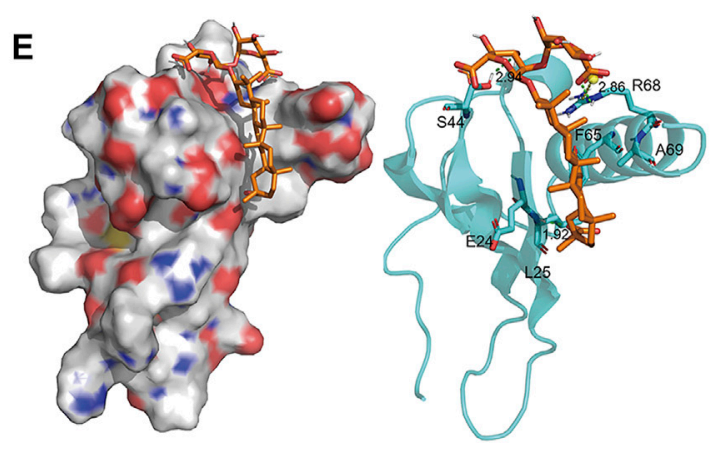

FIGURE 5 | Docking conformation of five key components and their best combined target proteins. (A) 17-beta-estradiol bound best to MAPK1. It could form a hydrogen bond with Gly230 in MAPK1 and p- $\pi$ conjugated with His239. (B) Curcumin bound best to CXCL8. It could form three hydrogen bonds with Arg6, Lys11, and Glu48 in CXCL8, respectively. (C) Licochalcone A bound best to IL1B. It could form two hydrogen bonds with Met20 and Lys63 in IL1B, respectively. (D) Quercetin bound best to ILIB. It could form a hydrogen bond with Met20, two hydrogen bonds with Val41, and two hydrogen bonds with Lys63 in IL1B. (E) Glycyrrhizic acid bound best to CXCL8. It could form three hydrogen bonds with Leu25, Ser44, and Arg68 in CXCL8, respectively, and a salt bridge with Arg68.

could enter into the blood circulation via transdermal delivery. Thus, glycyrrhizic acid, curcumin, $17-\beta$-estradiol, licochalcone A, and quercetin were determined as the candidate key compounds of BMO. Studies have shown that the above compounds have significant anti-inflammatory effects and can act on related cytokines such as IL-1 $\beta$, TNF $\alpha$, and other targets, and the NF- $\kappa \mathrm{B}$ pathway plays a role in treating OA. Researches have shown that glycyrrhizic acid does well in anti-inflammatory and immunomodulatory effects. Naemehsadat $\mathrm{T}$ et al. demonstrated that glycyrrhizic acid could significantly decrease serum TNF- $\alpha$, IL-6, and IL- $1 \beta$ in overweight young men to decrease muscular damage (Tavvafian et al., 2020). Feng et al. (2013) found that glycyrrhizic acid could cause the inhibition of the RAGE/NF- $\kappa \mathrm{B}$ pathway, thus having anti-apoptotic, anti-inflammatory, and antioxidative stress effects, which was demonstrated to have a protective effect on AGEs-induced endothelial dysfunction. 17$\beta$-estradiol is the major component of estrogen, which was considered to have an association with developing knee OA in middle-aged women (Sowers et al., 2006). Huang et al. (2011) established an OA model in rats and cultured chondrocytes to investigate the function of 17- $\beta$-estradiol, which showed that 17 $\beta$-estradiol could regulate the PI3K/Akt pathway to promote cell proliferation in chondrocytes. It has been widely reported that curcumin has a significant therapeutic effect on OA by regulating cytokines such as IL6, TNF- $\alpha$, IL1B, and TNF, and signaling pathways such as IL-17 and NF-kB signaling pathways (Sylvester et al., 2004; Zhang and Zeng, 2019). Licochalcone A has a significant anti-inflammatory effect via regulating various pathways such as MAPK, NF- $\mathrm{B}$, and Wnt/beta-catenin signaling pathways (Chen et al., 2017; Lien et al., 2017). Yang et al. (2018) revealed that licochalcone A inhibited the progression and development of arthritis in vivo via suppressing NF- $\kappa \mathrm{B}$ activation. Jia et al. (2017) demonstrated that licochalcone A could activate the Nrf2 signaling pathway 


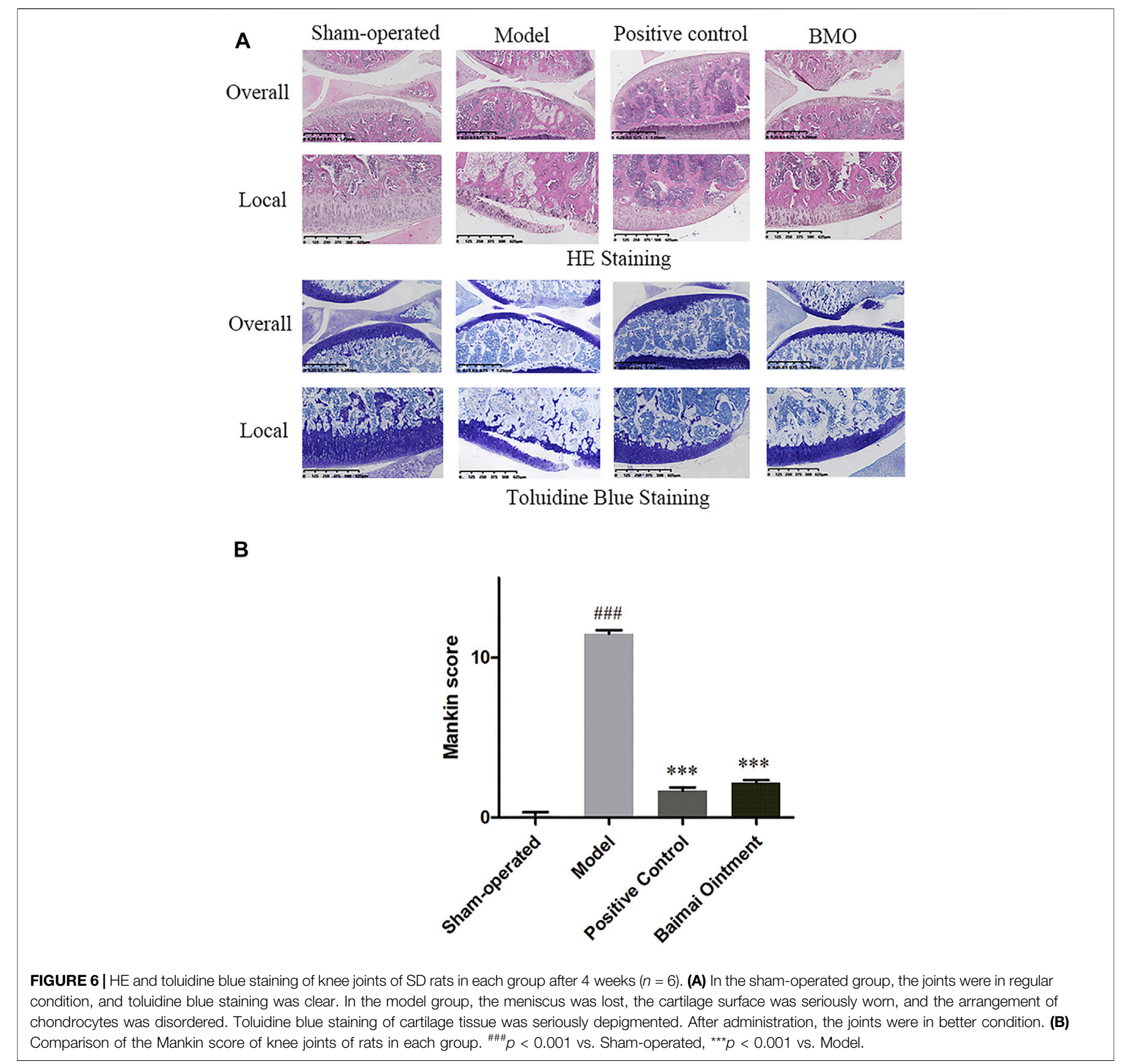

to show anti-inflammatory effects in rat chondrocytes stimulated by IL1 $\beta$. Quercetin has been reported to have effects on various OA animal models. Hu et al. (2019) revealed that quercetin had a great alleviation effect on the rat OA model via inhibiting inflammation and apoptosis of chondrocytes, having synovial macrophages polarized to M2 macrophages. Qiu et al. (2018) verified that quercetin upregulated the AMPK/SIRT1 signaling pathway to attenuate mitochondrial dysfunction and biogenesis in $\mathrm{OA}$ rats.

To obtain the potential targets of BMO in treating OA, we intersected the targets of BMO and OA and got a total of 169 overlapped targets. The PPI network was constructed to analyze the relationship between these overlapped target proteins.
Moreover, the higher degree value represents the more important part the target takes in. The targets with high degrees were IL6, TNF, MAPK1, VEGFA, CXCL8, and IL1B, and they were determined as key targets. Next, molecular docking has been conducted, and the binding energy of key compounds and key target proteins was low which indicated strong affinities between them. According to the results of animal experiments, $\mathrm{BMO}$ had a great therapeutic effect on $\mathrm{OA}$ in rats, and its mechanism for treating OA was mainly through regulating the key target proteins mentioned above. It has been reported that these targets are involved in inflammation, immunity, and apoptosis, which greatly contribute to the pathogenesis of OA. IL6, derived from macrophages, chondrocytes, and osteoclasts 

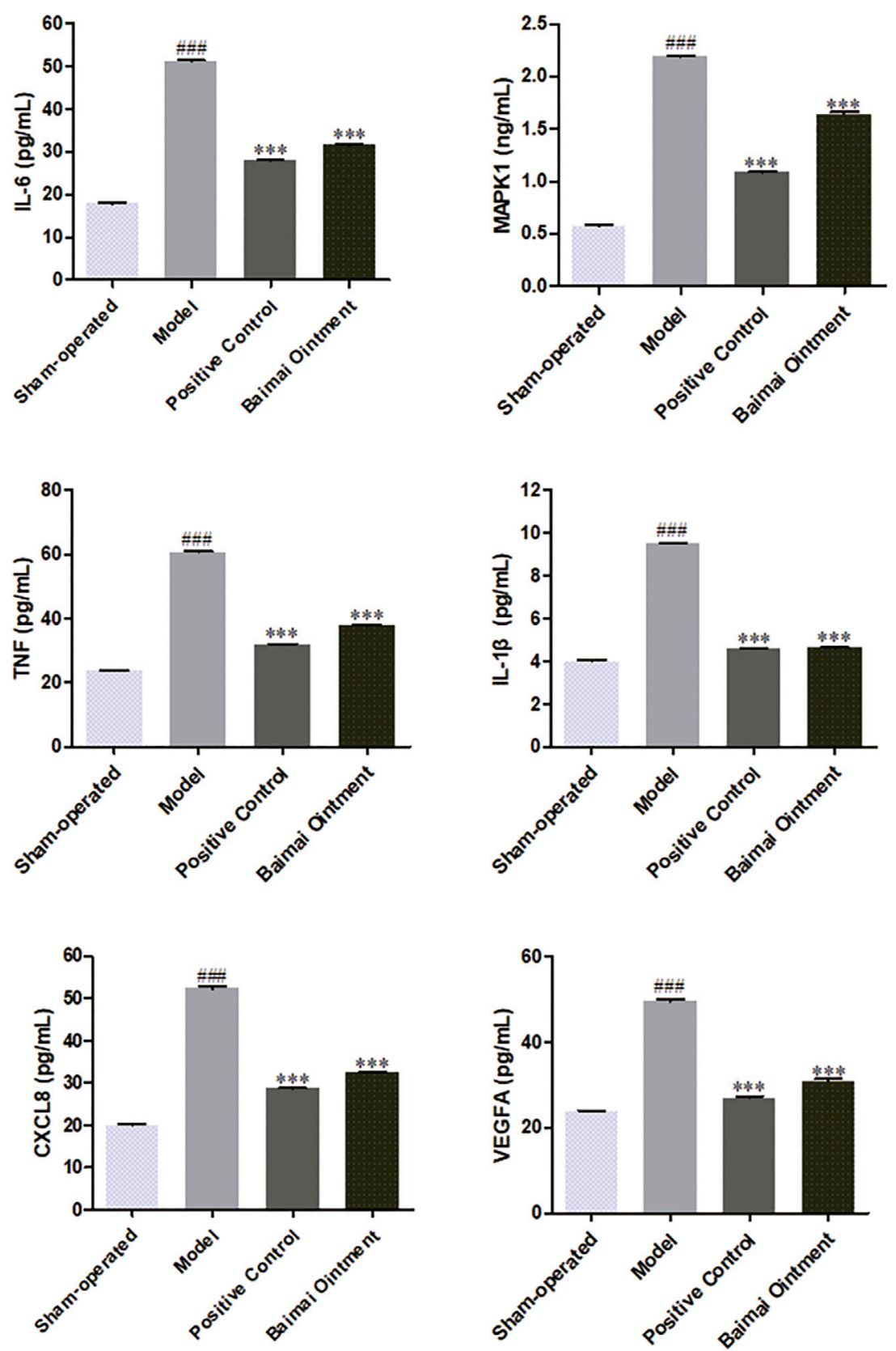

FIGURE 7| Expression of IL-6, MAPK1, TNF, IL-1, CXCL8, and VEGFA proteins in the joint fluid of rats in each group after 4 weeks $(n=6)$. \#\#\# $p<0.001$ vs. Shamoperated, ${ }^{\star \star \star} p<0.001$ vs. Model.

can increase inflammatory cells in synovial tissue and stimulate chondrocyte proliferation. Dewa GK et al. found that the incidence of lumbar OA in patients with high levels of IL6 was five times higher than in patients with low levels of IL6, suggesting that IL6 is closely related to the occurrence of OA (Pratama et al., 2019). TNF, derived from macrophages and chondrocytes, serves as an indispensable mediator in the degradation of the cartilage matrix and is closely related to synovitis, and it has been widely reported that many drugs interact with TNF to make an effect on OA. Yuan et al.
(2010) probed into the mechanism of Juanbi Capsules in guarding against knee $\mathrm{OA}$ in the rabbit and found it possible to decrease serum TNF- $\alpha$, IL-1, and IL- 6 contents to prevent OA. MAPK family is a group of evolutionarily conserved serine/ threonine kinases, which can regulate inflammation and apoptosis. Studies have shown that BMO can modify the expression of MAPK (Guo et al., 2009). The increased level of VEGFA is related to the progression of OA. It is involved in the special pathological process of $\mathrm{OA}$, including cartilage degeneration, osteophyte formation, subchondral bone cysts 


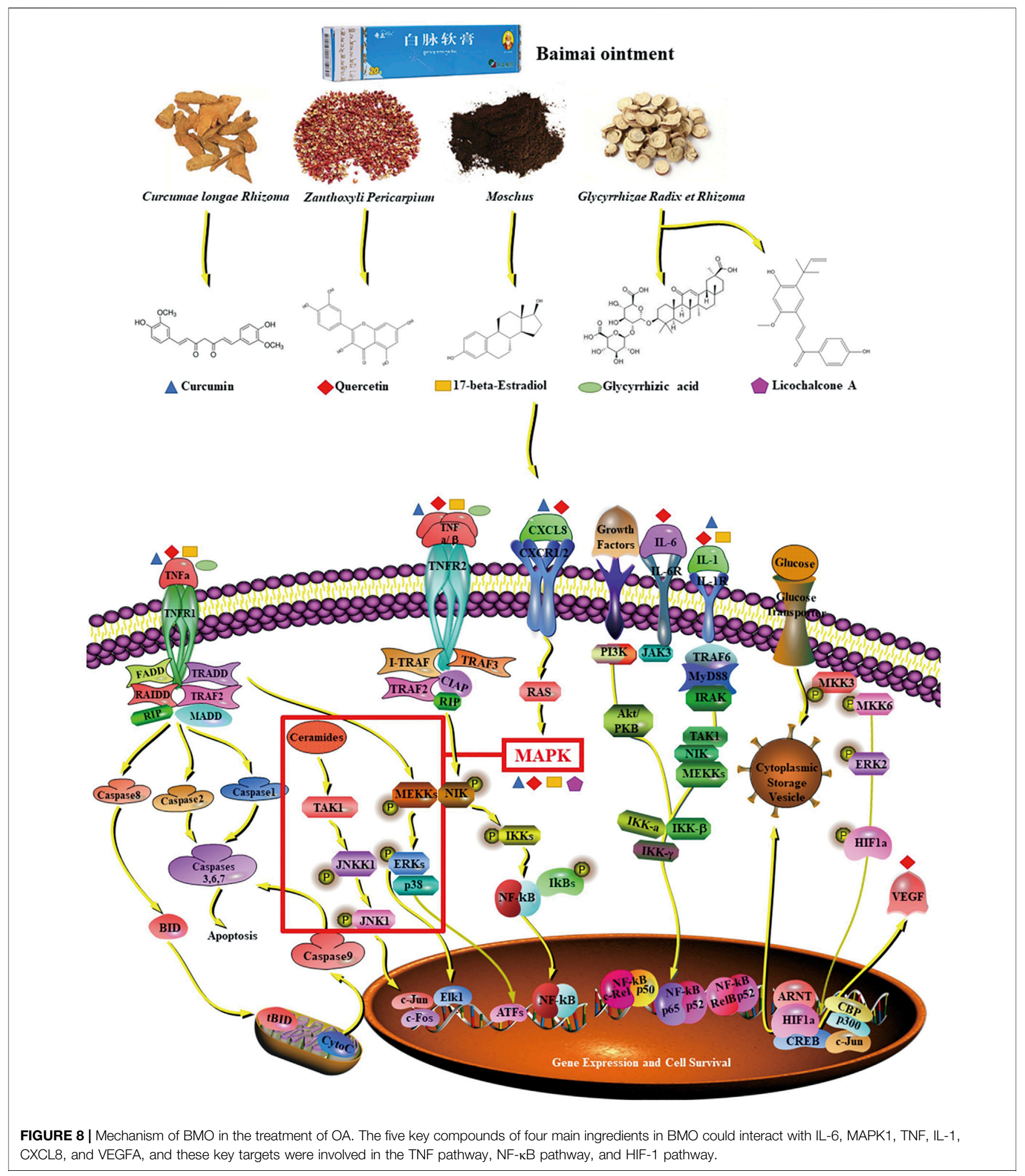

and sclerosis, synovitis, and pain (Nelson, 2017). Inhibition of VEGFA signal transduction can reduce the progression of OA (Hamilton et al., 2016). Research has shown that IL1 $\beta$ expression could increase by advanced oxidation protein products in rat chondrocytes, then the progress of cartilage degeneration accelerated in OA, which illustrated the relationship between 
IL-1 $\beta$ and OA (Liao et al., 2020). Yang et al. (2016) found that CXCL8 and CXCL11 could accelerate cell apoptosis and suppress chondrocytes proliferation, which is believed to be related to the regulation of the JAK-STAT signaling pathway as well as the NF$\kappa \mathrm{B}$ and MAPK pathways and the enhancement of other proinflammatory cytokines expressions. Therefore, CXCL8/11 may exacerbate the disease progression of OA, and it may be designed as a new therapeutic target for OA.

2082 GO terms and 159 KEGG pathways have been obtained by GO and KEGG enrichment. After analyzing each pathway, it showed that BMO could regulate immune function and inhibit inflammatory reactions through inflammatory, immune, and cellular metabolism pathways, so as to achieve clinical therapeutic effects on OA. The representative pathways include TNF, NF- $\kappa \mathrm{B}$, and HIF-1 pathways. The TNF signaling pathway is mediated by related inflammatory factors such as TNF- $\alpha$, which mainly play the role of regulating inflammation. There are many genes related to inflammation in the NF- $\kappa \mathrm{B}$ pathway, so it has chronic activity in many inflammatory diseases, especially, in OA. The NF- $\kappa \mathrm{B}$ pathway is closely associated with the pathophysiology of OA via a variety of effects and can be activated in OA chondrocytes in the course of aging and inflammation (Marcu et al., 2010; Saito, 2020). Wu et al. (2020) demonstrated that stachydrine protected chondrocytes from IL-1 $\beta$-induced inflammation through the the NF- $\kappa B$ pathway. Similarly, the HIF-1 pathway involves a transcription factor in the basic helix-ring-helix-PAS domain, which is very essential in response to hypoxia. Studies have shown that upregulation of HIF-2a contributes to the progression of OA via inducing primary cilia loss, also it might be designed as therapeutic targets for OA (Yang et al., 2019). Li et al. (2020) found that knee OA induced by monoiodoacetic acid could be suppressed via inhibiting HIF-1 $\alpha$ /NLRP3 inflammasome signaling.

The current study exhibits several limitations though. Firstly, the network pharmacology method is based on the existing research results to explore the mechanism of the prescription. There may be a small number of pharmaceutical ingredients and action targets that have not been reported yet, and OA-related targets are also constantly updated, so this study may not be sufficient. In addition, oral bioavailability $\geq 30 \%$ and DL $\geq 0.18$ were normally used as screening criteria in many studies. In this study, BMO is an external preparation, then the screening criterion was only $\mathrm{DL} \geq 0.18$, which may omit some active compounds (e.g., the DL of glycyrrhizic acid is 0.11). Lastly, although molecular docking technology has been widely used in various drug research fields, it still has some limitations just as a computer virtual screening method. For example, quercetin and cholesteryl ferulate, the main components of BMO screened in network pharmacology, had high binding energy with targets, which meant difficulty in combining. It may be because the target is not fixed and may change with the environment in vivo, which cannot be simulated by molecular docking. In this study, the combination of the two methods to explore the molecular mechanism of BMO in the treatment of OA and experimental validation was necessary to overcome the limitations to a certain extent.

\section{CONCLUSION}

In this study, it was found that $\mathrm{BMO}$ had multicomponents and multi-targets in treating OA. The possible molecular mechanism of action is that the candidate key components of glycyrrhizic acid, 17- $\beta$-estradiol, curcumin, licochalcone $\mathrm{A}$, and quercetin act on IL6, TNF, MAPK1, VEGFA, CXCL8, and IL1B and these key targets regulate the TNF signaling pathway, NF- $\kappa B$ signaling pathway, and HIF-1 signaling pathway, so as to play a therapeutic role (Figure 8). The verification of molecular docking and pharmacological experiments demonstrated that the key components and key target proteins obtained from network pharmacology research combined well. The result of the network pharmacology study is reliability, and it is reasonable to explain the mechanism of BMO in treating OA. The study demonstrated that $\mathrm{BMO}$ had a great therapeutic effect on OA in the rat model for the first time, which could partly recover the lesion of joints and significantly decrease cytokines such as CXCL8, IL-1 $\beta$, IL-6, MAPK1, TNF, and VEGFA to alleviate inflammation.

Traditional medicine is a valuable source for disease treatment, such as Tibetan medicine has abundant applications in the clinic. Hence, to study its mechanism for treatment is necessary. However, its molecular mechanism is difficult to reveal because of complicated components. This study combined network pharmacology and molecular docking methods with pharmacological experiments to uncover the molecular mechanism of Tibetan medicine BMO in treating OA from three aspects of components, targets, and pathways. The strategy we adopted was both reliable and efficient to study the molecular mechanism of external preparation with a combination of theoretical pharmacology and experimental pharmacology.

The significance of this study is preliminarily clarifying the molecular mechanism of $\mathrm{BMO}$ in treating $\mathrm{OA}$, thus providing theoretical foundation for clinical application and further research. The obtained candidate key components may be designed for new drugs due to their great effects. Also, the key targets and potential pathways selected can provide new ideas and directions for targeted intervention therapy in treating $\mathrm{OA}$ in future development. With the progress of bioinformatic technologies such as molecular fishing, molecular dynamics simulation, and so on, the study of molecular mechanism and drug design has been efficient. Later, binding with advanced bioscience techniques such as DNA chips, single-cell sequence, and surface plasmon resonance can be an effective verification. The theoretical-experimental research pattern may serve as a universal method in drug study.

\section{DATA AVAILABILITY STATEMENT}

The original contributions presented in the study are included in the article/Supplementary Material; further inquiries can be directed to the corresponding authors. 


\section{ETHICS STATEMENT}

The animal study was reviewed and approved by the Animal Ethics Committee of Beijing University of Chinese Medicine.

\section{AUTHOR CONTRIBUTIONS}

YZ, SD, and HW contributed to conceptualization; SD contributed to funding acquisition; YZ, WZ, and JP contributed to investigation; $\mathrm{YZ}, \mathrm{WZ}$, and $\mathrm{HW}$ contributed to methodology; SD and HW contributed to supervision; YZ, SD, and HW contributed to writing, review, and editing.

\section{REFERENCES}

Buhrmann, C., Mobasheri, A., Busch, F., Aldinger, C., Stahlmann, R., Montaseri, A., et al. (2011). Curcumin Modulates Nuclear Factor $\kappa B$ (NF-Kb)-Mediated Inflammation in Human Tenocytes In Vitro. J. Biol. Chem. 286, 28556-28566. doi:10.1074/jbc.M111.256180

Centers for Disease Control and Prevention (2020). Osteoarthritis (OA). Available at: https://www.cdc.gov/arthritis/basics/osteoarthritis.htm (Accessed July 27, 2020).

Chen, M., and Sun, Q. (2021). Systemic Pharmacology Understanding of the Key Mechanism of Sedum Sarmentosum Bunge in Treating Hepatitis. Naunynschmiedeberg's Arch. Pharmacol. 394, 421-430. doi:10.1007/s00210-02001952-9

Chen, W.-P., Hu, Z.-N., Jin, L.-B., and Wu, L.-D. (2017). Licochalcone A Inhibits MMPs and ADAMTSs via the NF-Kb and Wnt/ $\beta$-Catenin Signaling Pathways in Rat Chondrocytes. Cell Physiol Biochem. 43, 937-944. doi:10.1159/ 000481645

Cui, Q., Zhang, Y.-l., Ma, Y.-h., Yu, H.-y., Zhao, X.-z., Zhang, L.-h., et al. (2020). A Network Pharmacology Approach to Investigate the Mechanism of Shuxuening Injection in the Treatment of Ischemic Stroke. J. Ethnopharmacology. 257, 112891. doi:10.1016/j.jep.2020.112891

Dingzeng, N. M., Xiang, Y., Qiangba, D. Z., and Yan, H. T. (2020). Comparative Effect of Baimai Ointment in Multimodal Analgesia After Knee Arthroplasty. Integrated traditional Chin. West. Med. Cardiovasc. Dis. 8 (06), 151. doi:10.16282/j.cnki.cn11-9336/r.2020.06.148

Feng, L., Zhu, M.-m., Zhang, M.-h., Wang, R.-s., Tan, X.-b., Song, J., et al. (2013). Protection of Glycyrrhizic Acid against AGEs-Induced Endothelial Dysfunction through Inhibiting RAGE/NF- $\mathrm{kB}$ Pathway Activation in Human Umbilical Vein Endothelial Cells. J. Ethnopharmacology. 148, 27-36. doi:10.1016/j.jep.2013.03.035

Ferreira, L., Dos Santos, R., Oliva, G., and Andricopulo, A. (2015). Molecular Docking and Structure-Based Drug Design Strategies. Molecules. 20, 13384-13421. doi:10.3390/molecules200713384

Fortin, P. R., Penrod, J. R., Clarke, A. E., St-Pierre, Y., Joseph, L., Bélisle, P., et al. (2002). Timing of Total Joint Replacement Affects Clinical Outcomes Among Patients With Osteoarthritis of the Hip or Knee. Arthritis Rheum. 46, 3327-3330. doi:10.1002/art.10631

Fu, K., Xu, M., Zhou, Y., Li, X., Wang, Z., Liu, X., et al. (2020). The Status Quo and Way Forwards on the Development of Tibetan Medicine and the Pharmacological Research of Tibetan Materia Medica. Pharmacol. Res. 155, 104688. doi:10.1016/j.phrs.2020.104688

Gong, Z., Liu, S. D., Jiang, C. M., and Li, D. H. (2012). The Efficiency and Safety Evaluation of Baimai Ointment on Alleviating Hypermyotonia Following Cerebral Infarction. Mod. Med. J. China. 14 (01), 47-49. doi:10.3969/ j.issn.1672-9463.2012.01.018

Guo, W. H., Zhang, W. W., Huang, Y. H., and Li, Y. (2009). Effect of Baimai Ointment on the Expression of p38MAPK and GAP-43 in Ischemic Brain of Rats. J. Beijing Med. 31 (01), 41-44. doi:10.3969/j.issn.1008-4118.2007.02.024

\section{FUNDING}

This work was financially supported by the Scientific Research Project of Chinese National Medical Association (No. 2019KYXMM101-21) and Special Funds for Fundamental Research Expenses of Central Universities (2020-JYB-ZDGG-024), the National Natural Science Foundation of China (Grant No. 82104545).

\section{SUPPLEMENTARY MATERIAL}

The Supplementary Material for this article can be found online at: https://www.frontiersin.org/articles/10.3389/fgene.2021.750681/ full\#supplementary-material

Hamilton, J. L., Nagao, M., Levine, B. R., Chen, D., Olsen, B. R., and Im, H.-J. (2016). Targeting VEGF and its Receptors for the Treatment of Osteoarthritis and Associated Pain. J. Bone Miner Res. 31, 911-924. doi:10.1002/jbmr.2828

Hu, Y., Gui, Z., Zhou, Y., Xia, L., Lin, K., and Xu, Y. (2019). Quercetin Alleviates Rat Osteoarthritis by Inhibiting Inflammation and Apoptosis of Chondrocytes, Modulating Synovial Macrophages Polarization to M2 Macrophages. Free Radic. Biol. Med. 145, 146-160. doi:10.1016/j.freeradbiomed.2019.09.024

Huang, J. G., Xia, C., Zheng, X. P., Yi, T. T., Wang, X. Y., Song, G., et al. (2011). 17ßEstradiol Promotes Cell Proliferation in Rat Osteoarthritis Model Chondrocytes via PI3K/Akt Pathway. Cell Mol Biol Lett. 16, 564-575. doi:10.2478/s11658-011-0023-y

Jia, T., Qiao, J., Guan, D., and Chen, T. (2017). Anti-Inflammatory Effects of Licochalcone A on IL-1 $\beta$-Stimulated Human Osteoarthritis Chondrocytes. Inflammation. 40, 1894-1902. doi:10.1007/s10753-017-0630-5

Jiang, D., Ding, D. F., and Han, D. P. (2019). Effects of Plasma Containing Baimai Ointment on Co-cultivation of Rat Degenerative Synoviocytes and Chondrocytes and its Mechanism. J. Guangzhou Univ. Traditional Chin. Med. 36 (04), 556-562. doi:10.13359/j.cnki.gzxbtcm.2019.04.023

Kim, W. J., Yang, S., Choi, G., Park, I., Noh, P., Seo, C. S., et al. (2019). Development of Conventional PCR and Real-time PCR Assays to Discriminate the Origins of Chinese Pepper Oil and Herbal Materials From Zanthoxylum. J. Sci. Food Agric. 99, 2021-2029. doi:10.1002/jsfa.9458

Kolasinski, S. L., Neogi, T., Hochberg, M. C., Oatis, C., Guyatt, G., Block, J., et al. (2020). 2019 American College of Rheumatology/Arthritis Foundation Guideline for the Management of Osteoarthritis of the Hand, Hip, and Knee. Arthritis Care Res. 72, 149-162. doi:10.1002/acr.24131

Krustev, E., Rioux, D., and McDougall, J. J. (2015). Mechanisms and Mediators that Drive Arthritis Pain. Curr. Osteoporos. Rep. 13, 216-224. doi:10.1007/s11914015-0275-y

Lane, N. E., and Thompson, J. M. (1997). Management of Osteoarthritis in the Primary-Care Setting: an Evidence-Based Approach to Treatment. Am. J. Med. 103 (6a), 25S-30S. doi:10.1016/s0002-9343(97)90005-x

Li, S., Xue, X., Yang, X., Zhou, S., Wang, S., and Meng, J. (2019). A Network Pharmacology Approach Used to Estimate the Active Ingredients of Moutan Cortex Charcoal and the Potential Targets in Hemorrhagic Diseases. Biol. Pharm. Bull. 42, 432-441. doi:10.1248/bpb.b18-00756

Li, X., Mei, W., Huang, Z., Zhang, L., Zhang, L., Xu, B., et al. (2020). Casticin Suppresses Monoiodoacetic Acid-Induced Knee Osteoarthritis Through Inhibiting HIF-1 $\alpha /$ NLRP3 Inflammasome Signaling. Int. Immunopharmacology. 86, 106745. doi:10.1016/j.intimp.2020.106745

Liang, J., Wu, H. C., Du, S. Y., Liu, C. F., Zhong, L. Y., Zhang, Q., et al. (2019). In Vitro Transdermal Permeation of Main Compositions in Baimai Ointment. Zhongguo Zhong Yao Za Zhi. 44, 2486-2492. doi:10.19540/ j.cnki.cjcmm.20190221.008

Liao, C.-R., Wang, S.-N., Zhu, S.-Y., Wang, Y.-Q., Li, Z.-Z., Liu, Z.-Y., et al. (2020). Advanced Oxidation Protein Products Increase TNF- $\alpha$ and IL-1 $\beta$ Expression in Chondrocytes via NADPH Oxidase 4 and Accelerate Cartilage Degeneration in Osteoarthritis Progression. Redox Biol. 28, 101306. doi:10.1016/ j.redox.2019.101306 
Lien, L. M., Lin, K. H., Huang, L. T., Tseng, M. F., Chiu, H. C., Chen, R. J., et al. (2017). Licochalcone A Prevents Platelet Activation and Thrombus Formation through the Inhibition of PLC $\gamma 2$-PKC, Akt, and MAPK Pathways. Int. J. Mol. Sci. 18 (7), 1500. doi:10.3390/ijms 18071500

Lipinski, C. A., Lombardo, F., Dominy, B. W., and Feeney, P. J. (2001). Experimental and Computational Approaches to Estimate Solubility and Permeability in Drug Discovery and Development Settings. Adv. Drug Deliv. Rev. 46 (1), 3-26. doi:10.1016/s0169-409x(00)00129-0

Liu, J., Shao, H., Fang, S., Cheng, Y., Ling, P., and Chen, J. (2019). Evaluation of Pharmacokinetics and Pharmacodynamics of Sinomenine-Hyaluronic Acid Conjugate After Intra-Articular Administration for Osteoarthritis Treatment. Drug. Des. Devel. Ther. 13, 657-665. doi:10.2147/dddt.S186558

Liu, S. D., Jiang, C. M., Li, D. H., Xie, C. P., and Yan, J. X. (2007). Efficiency and Safety Evaluation of Baimai Ointment for Alleviating Hypermyotonia Following Cerebral Infarction. Neural Regen. Res. 10, 607-610. CNKI:SUN: SJZY.0.2007-10-009

Mankin, H. J., Johnson, M. E., and Lippiello, L. (1981). Biochemical and Metabolic Abnormalities in Articular Cartilage from Osteoarthritic Human Hips. III. Distribution and Metabolism of Amino Sugar-Containing Macromolecules. J. Bone Jt. Surg. 63, 131-139. doi:10.2106/00004623-198163010-00017

Marcu, K. B., Otero, M., Olivotto, E., Maria Borzi, R., and B. Goldring, M. (2010). NF-кB Signaling: Multiple Angles to Target OA. Curr. Drug. Targets. 11, 599-613. doi:10.2174/138945010791011938

Nelson, F. (2017). Circulation in Rapid Hip and Knee Bone Destruction. J. Hip Surg. 1 (1), 055-060. doi:10.1055/s-0037-1602181

Nüesch, E., Dieppe, P., Reichenbach, S., Williams, S., Iff, S., Jüni, P., et al. (2011). All Cause and Disease Specific Mortality in Patients With Knee or Hip Osteoarthritis: Population Based Cohort Study. Bmj 342, d1165. doi:10.1136/bmj.d1165

Pang, X.-C., Kang, D., Fang, J.-S., Zhao, Y., Xu, L.-J., Lian, W.-W., et al. (2018). Network Pharmacology-Based Analysis of Chinese Herbal Naodesheng Formula for Application to Alzheimer's Disease. Chin. J. Nat. Medicines. 16, 53-62. doi:10.1016/s1875-5364(18)30029-3

Petrella, R., and Wakeford, C. (2015). Pain Relief and Improved Physical Function in Knee Osteoarthritis Patients Receiving Ongoing Hylan G-F 20, a HighMolecular-Weight Hyaluronan, versus Other Treatment Options: Data From a Large Real-World Longitudinal Cohort in Canada. Drug. Des. Devel. Ther. 9, 5633-5640. doi:10.2147/dddt.S88473

Pratama, D. G. K., Suyasa, I. K., Astawa, P., and Lestari, A. A. W. (2019). High IL-6 Level as a Marker of Lumbar Osteoarthritis in Patients Older Than 55 Years with Low Back Pain. Orthop. Res. Rev. 11, 17-21. doi:10.2147/orr.S188678

Qiu, L., Luo, Y., and Chen, X. (2018). Quercetin Attenuates Mitochondrial Dysfunction and Biogenesis via Upregulated AMPK/SIRT1 Signaling Pathway in OA Rats. Biomed. Pharmacother. 103, 1585-1591. doi:10.1016/ j.biopha.2018.05.003

Ren, X. Q., Ren, Q. J., and Zheng, L. J. (2018). Clinical Application Analysis and Popularization of Baimai Disease Therapy. World Sci. Technol. modernization traditional Chin. Med. 20 (03), 453-459.

Saito, T. (2020). NF-kappaB and HIF Signaling in Osteoarthritis. Encyclopedia Bone Biol., 605-608. doi:10.1016/B978-0-12-801238-3.62214-6

Sellam, J., Courties, A., Eymard, F., Ferrero, S., Latourte, A., Ornetti, P., et al. (2019). Recommendations of the French Society of Rheumatology for the Pharmacological Management of Knee Osteoarthritis. Osteoarthritis and Cartilage. 27, S507-S508. doi:10.1016/j.joca.2019.02.573

Sowers, M. R., McConnell, D., Jannausch, M., Buyuktur, A. G., Hochberg, M., and Jamadar, D. A. (2006). Estradiol and its Metabolites and Their Association With Knee Osteoarthritis. Arthritis Rheum. 54, 2481-2487. doi:10.1002/art.22005

Sylvester, J., Liacini, A., Li, W. Q., and Zafarullah, M. (2004). Interleukin-17 Signal Transduction Pathways Implicated in Inducing Matrix Metalloproteinase-3, -13 and Aggrecanase-1 Genes in Articular Chondrocytes. Cell Signal. 16, 469-476. doi:10.1016/j.cellsig.2003.09.008

Tao, X.-M., Liu, P.-F., Gu, H.-Y., Lian, D.-B., Gao, L., Tao, W.-W., et al. (2020). Cordycepin Alleviates Anterior Cruciate Ligament Transection (ACLT)Induced Knee Osteoarthritis through Regulating TGF- $\beta$ Activity and Autophagy [Retraction]. Drug. Des. Devel. Ther. 14, 4133-4134. doi:10.2147/ dddt.S283594
Tavvafian, N., Darabi, H., Ahani, A., Naghizadeh, H., Hajiaghaee, R., RahmatiAhmadabad, S., et al. (2020). Effects of Glycyrrhizic Acid Supplementation during Nonlinear Resistance Training on Inflammatory Markers and Muscular Damage Indices in Overweight Young Men - ScienceDirect. Obes. Med. 17, 1. doi:10.1016/j.obmed.2019.100178

Totlis, T., Marín Fermín, T., and Kalifis, G. (2021). Arthroscopic Debridement for Focal Articular Cartilage Lesions of the Knee: A Systematic Review. Surgeon. doi:10.1016/j.surge.2020.11.011

von Mering, C., Jensen, L. J., Snel, B., Hooper, S. D., Krupp, M., Foglierini, M., et al. (2005). STRING: Known and Predicted Protein-Protein Associations, Integrated and Transferred across Organisms. Nucleic Acids Res. 33, D433-D437. doi:10.1093/nar/gki005

Wang, W. M., and Li, B. Y. (2019). Therapeutic Effect of Acupuncture Combined With Baimai Ointment on Knee Osteoarthritis of Cold Dampness Obstruction Type. Strait Pharm. J. 31 (12), 123-125.

Wang, X., Wei, S., Liu, T., Pang, J., Gao, N., Ding, D., et al. (2014). Effectiveness, Medication Patterns, and Adverse Events of Traditional Chinese Herbal Patches for Osteoarthritis: a Systematic Review. Evid. Based Complement. Alternat Med. 2014, 343176. doi:10.1155/2014/343176

Wang, Y., Yu, W., Shi, C., Jiao, W., Li, J., Ge, J., et al. (2019). Network Pharmacology of Yougui Pill Combined with Buzhong Yiqi Decoction for the Treatment of Sexual Dysfunction. Evid. Based Complement. Alternat Med. 2019, 1243743. doi:10.1155/2019/1243743

Wang, Y., Zheng, C., Huang, C., Li, Y., Chen, X., Wu, Z., et al. (2015). Systems Pharmacology Dissecting Holistic Medicine for Treatment of Complex Diseases: An Example Using Cardiocerebrovascular Diseases Treated by TCM. Evid. Based Complement. Alternat Med. 2015, 980190. doi:10.1155/2015/980190

Wu, H., Zhang, M., Li, W., Zhu, S., and Zhang, D. (2020). Stachydrine Attenuates IL-1 $\beta$-induced Inflammatory Response in Osteoarthritis Chondrocytes Through the NF-Kb Signaling Pathway. Chemico-Biological Interactions. 326, 109136. doi:10.1016/j.cbi.2020.109136

Xing-ping, Z., Gen-rong, X., Shan-qiang, X., Ze-ming, L., and Lei, H. (2014). Casecontrol Study on Tibetan Baimai Ointment for the Treatment of WristDysfunction After Distal Radius Fracture. Zhongguo gu shang = China J. orthopaedics Traumatol. 27 (11), 920-924. doi:10.3969/j.issn.10030034.2014.11.009

Xu, Q., Chen, B., Wang, Y., Wang, X., Han, D., Ding, D., et al. (2017). The Effectiveness of Manual Therapy for Relieving Pain, Stiffness, and Dysfunction in Knee Osteoarthritis: A Systematic Review and Meta-Analysis. Pain Physician. 20, 229-243. doi:10.36076/ppi.2017.243

Yan, C., and Zhou, Z. (2021). Ellagic Acid and Pentagalloylglucose Are Potential Inhibitors of Prion Protein Fibrillization. Int. J. Biol. Macromolecules. 172, 371-380. doi:10.1016/j.ijbiomac.2021.01.045

Yang, F., Su, X., Pi, J., Liao, K., Zhou, H., Sun, Y., et al. (2018). Atomic Force Microscopy Technique Used for Assessment of the Anti-Arthritic Effect of Licochalcone A via Suppressing NF-Kb Activation. Biomed. Pharmacother. 103, 1592-1601. doi:10.1016/j.biopha.2018.04.142

Yang, H., Zhang, W., Huang, C., Zhou, W., Yao, Y., Wang, Z., et al. (2014). A Novel Systems Pharmacology Model for Herbal Medicine Injection: a Case Using Reduning Injection. BMC Complement. Altern. Med. 14, 430. doi:10.1186/1472-6882-14-430

Yang, P., Tan, J., Yuan, Z., Meng, G., Bi, L., and Liu, J. (2016). Expression Profile of Cytokines and Chemokines in Osteoarthritis Patients: Proinflammatory Roles for CXCL8 and CXCL11 to Chondrocytes. Int. Immunopharmacology. 40, 16-23. doi:10.1016/j.intimp.2016.08.005

Yang, Q., Zhou, Y., Cai, P., Fu, W., Wang, J., Wei, Q., et al. (2019). Up-regulated HIF-2a Contributes to the Osteoarthritis Development Through Mediating the Primary Cilia Loss. Int. Immunopharmacology. 75, 105762. doi:10.1016/ j.intimp.2019.105762

Yu, M.-X., Ma, X.-Q., Song, X., Huang, Y.-M., Jiang, H.-T., Wang, J., et al. (2020). Validation of the Key Active Ingredients and Anti-Inflammatory and Analgesic Effects of Shenjin Huoxue Mixture Against Osteoarthritis by Integrating Network Pharmacology Approach and Thin-Layer Chromatography Analysis. Drug Des Devel Ther. 14, 1145-1156. doi:10.2147/dddt.S243951

Yuan, P.-w., Liu, D.-y., Chu, X.-d., Hao, Y.-q., Zhu, C., and Qu, Q. (2010). Effects of Preventive Administration of Juanbi Capsules on TNF- $\alpha$ IL-1 and IL-6 Contents of Joint Fluid in the Rabbit With Knee Osteoarthritis. J. Traditional Chin. Med. 30, 254-258. doi:10.1016/s0254-6272(10)60052-0 
Zeng, Y., Lou, G., Ren, Y., Li, T., Zhang, X., Wang, J., et al. (2021). Network Pharmacology-Based Analysis of Zukamu Granules for the Treatment of COVID-19. Eur. J. Integr. Med. 42, 101282. doi:10.1016/j.eujim.2020.101282

Zhang, W. Q., Yang, L. P., Song, Q. H., et al. (2012). Effect of Baimai Ointment on Fracture Healing Process and Osteotylus Tissues in Rabbit Model of Radial Fracture. Chin. J. Basic Med. Traditional Chin. Med. 18 (02), 170-172. CNKI:SUN:ZYJC.0.2012-02-023

Zhang, Y., and Zeng, Y. (2019). Curcumin Reduces Inflammation in Knee Osteoarthritis Rats Through Blocking TLR4/MyD88/NF-кB Signal Pathway. Drug Dev. Res. 80, 353-359. doi:10.1002/ddr.21509

Zhang, Z., Zhou, S. J., Chen, L. J., Tian, J., and Chen, W. W. (2018). A Brief Account of Tibetan Medicine White Vein Theory and the Research Progress of Baimai Ointment in the Past 10 Years. J. Med. Pharm. Chin. Minorities. 24 (11), 63-66. doi:10.3969/j.issn.1006-6810.2018.11.027

Zhou, X., Zhang, L., Guo, X., Liu, G., Wang, G., and Fu, S. (2018). A Macaca Fascicularis Knee Osteoarthritis Model Developed by Modified Hulth Combined with Joint Scratches. Med. Sci. Monit. 24, 3393-3404. doi:10.12659/msm.906626
Conflict of Interest: The authors declare that the research was conducted in the absence of any commercial or financial relationships that could be construed as a potential conflict of interest.

Publisher's Note: All claims expressed in this article are solely those of the authors and do not necessarily represent those of their affiliated organizations, or those of the publisher, the editors, and the reviewers. Any product that may be evaluated in this article, or claim that may be made by its manufacturer, is not guaranteed or endorsed by the publisher.

Copyright $\odot 2021 \mathrm{Zhu}$, Zhong, Peng, $W u$ and Du. This is an open-access article distributed under the terms of the Creative Commons Attribution License (CC BY). The use, distribution or reproduction in other forums is permitted, provided the original author(s) and the copyright owner(s) are credited and that the original publication in this journal is cited, in accordance with accepted academic practice. No use, distribution or reproduction is permitted which does not comply with these terms. 